\title{
Considerações Gerais Acerca da Proposta de Teofrasto para o Estudo da Personalidade
}

\author{
General Considerations About Theophrastus Proposal in Personality Study \\ Consideraciones General em Teofrasto Propuesta para el Estudio de la Personalidad
}

Lucas de Francisco Carvalho ${ }^{1}$

Rodolfo Augusto Matteo Ambiel

Universidade São Francisco

\begin{abstract}
Resumo
A personalidade tem sido compreendida como padrões de características mais marcantes e estáveis ao longo do tempo e representa um dos construtos mais estudados na Psicologia. Dessa forma, o objetivo deste artigo é apresentar brevemente dados históricos a respeito do estudo da personalidade. São abordados os conceitos elementares do construto, bem como algumas inconsistências a respeito de sua conceituação atual. Além disso, são retomadas as raízes etimológicas e históricas do construto a partir do ponto de vista filosófico ao abordar o livro Os Caracteres de Toefrasto, filsósofo grego pré-socrático que viveu entre 372 a.C. e 287 a.C. São feitas considerações a respeito da influência que esse escrito têm no estudo da personalidade até os dias atuais. Conclui-se que, apesar de pouco citada dentre os precursores do estudo da personalidade, a obra de Teofrasto exerce grande influência atualmente na área. Palavras-Chave: Personalidade; filosofia; história da psicologia.
\end{abstract}

\begin{abstract}
Personality has been understood as patterns of the most striking and stable features over time and represents one of the most studied constructs in psychology. Thus, the aim of this paper is to briefly present the historical data regarding the study of personality. It includes the basic concepts about the construct, as well as some inconsistencies regarding its current conceptualization. Moreover, it was took over the etymological and historical construct roots from the philosophical point of view by approaching the book The Characters of Toefrasto, pre-Socratic greek philosofer who lived between $372 \mathrm{BC}$ and $287 \mathrm{BC}$. Some considerations are made about the influence that this book have in the study of personality today. It's concluded that, although seldom mentioned among the forerunners of the study of personality, the work of Theophrastus currently has great influence in the area.

Keywords: Personality; philosofy; history of psychology.
\end{abstract}

\begin{abstract}
Resumen
La personalidad se ha entendido como los patrones de las características más llamativas y estable en el tiempo y representa una de las construcciones más estudiado en psicología. Así, el objetivo de este trabajo es presentar brevemente los datos históricos sobre el estudio de la personalidad. Incluye los conceptos básicos de el constructo, así como algunas inconsistencias en cuanto a su conceptualización actual. Ademas, hacerse cargo de las raíces etimológicas e históricas de construir desde el punto de vista filosófico para abordar el libro El carácter de Teofrasto, filsósofo griego presocrático que vivió entre 372 a. C. y 287 a. C. Se realizan algunas consideraciones acerca de la influencia que han escrito en el estudio de la personalidad a este día. Se concluye que, aunque poco mencionada entre los precursores del estudio de la personalidad, la obra de Teofrasto en la actualidad tiene una gran influencia en la área.
\end{abstract}

Palabras clave: Personalidad; filosofia; la historia de la psicologia.

\section{Introdução}

A psicologia pode ser compreendida como uma ciência que se propõe a estudar o comportamento do homem, os diversos domínios que o integram e suas relações. Não é raro que os profissionais que trabalham com definições e conceitos psicológicos se deparem com construtos de difícil acesso ou mensuração. Exemplo disso é a personalidade, cuja fonte de origem é o meio popular, tendo passado pela reflexão filosófica, e se configura como um amplo campo de estudo dentro da psicologia.

1 Endereço: Rua Alexandre Rodrigues Barbosa, 45, CEP 13251900, Itatiba, São Paulo, Fone (11) 45348118
Este trabalho configura-se no campo teórico, em uma tentativa de trazer à tona considerações acerca de um filósofo, Teofrasto, que de maneira mais ou menos direta contribuiu para o desenvolvimento posterior de modelos da personalidade. A partir disso, pretendeu-se discutir determinadas questões que permeiam as propostas contemporâneas para o estudo da personalidade.

\section{Personalidade}

De modo geral, a personalidade pode ser considerada como o estudo de conjuntos de características mais salientes e persistentes ao longo do tempo apresentadas por um indivíduo para lidar com 
as situações do cotidiano (Cloninger, 1999; ClapierValladon, 1988; Hall, Lindzey \& Campbell, 2000). Em outras palavras, a personalidade é frequentemente entendida como a combinação de diferentes sistemas relacionados aos atributos psicológicos (Allport, 1937; Mayer, 2005). Este texto tem o objetivo de apresentar, de maneira breve, dados históricos acerca do estudo da personalidade, antes mesmo de ser reconhecida como um construto psicológico, que deram base para as teorias atuais inseridas na psicologia.

No que concerne à etimologia do termo personalidade, tudo indica que seja indiretamente derivado da palavra etrusca phersu, que tinha a conotação de máscara e personagem (Poulsen, 1922). De maneira mais direta, sabe-se que a palavra personalidade tem suas raízes no latim, com o termo persona, que era usualmente utilizado no teatro greco-romano. Persona referia-se à máscara de teatro, que era utilizada pelos atores tanto para dar aparência (caracterização) de acordo com o personagem pretendido quanto para amplificar a voz para a platéia (Clapier-Valladon, 1988).

Nota-se que, ao longo dos anos, o sentido da palavra personalidade foi sendo modificado. No passado sugeria uma aparência pretendida, que caracterizaria um personagem, e, atualmente, é melhor entendida como o próprio indivíduo, suas características explícitas e implícitas, e a relação dessas características com o meio (Millon \& Davis, 1996; Smith, 1977). A despeito do significado subjacente ao termo, o estudo da personalidade sempre refletiu uma busca do ser humano para entender a natureza do seu próprio funcionamento (Oldham \& Morris, 1995).

Como apontam Hall e cols. (2000), são diversas as definições para personalidade, que podem tender para diferentes perspectivas. Apesar disso, esses autores alertam que nenhuma definição isolada da personalidade é completa e, portanto, não deve ser generalizada. A despeito da divergência sobre como pode ser melhor definida, há um consenso geral de que a personalidade é uma inferência abstrata, um conceito ou um construto, e não um fenômeno tangível (Millon, 1986a; 1993).

Segundo Oldham e Morris (1995), cada indivíduo possui um padrão próprio de funcionamento, que engloba pensamentos, sentimentos, atitudes, comportamentos, estratégias de enfrentamento, entre outros atributos. O funcionamento de cada indivíduo corresponde ao seu padrão de personalidade, que é uma amálgama de diferentes estilos separados e identificáveis.

Cada padrão de personalidade é único, como defendiam Allport e Allport (1921) e, por isso, a personalidade pode ser compreendida como uma face, isto é, não possui duplicatas, cada uma é uma mistura única de variados graus de diversos traços. Nesse sentido, no que concerne ao funcionamento psicológico, a personalidade pode ser considerada como o princípio organizador, aquilo que faz de cada indivíduo um ser singular (Millon \& Davis, 1996; Oldham \& Morris, 1995; Smith, 1977).

Pode-se dizer que os diversos autores consideram, em geral, a personalidade como o funcionamento de base e persistente ao longo do tempo no que respeita ao atributo psicológico de cada indivíduo. Nesse sentido, diferentes autores apontam para áreas gerais que fazem do estudo da personalidade algo imprescindível para o entendimento de cada indivíduo (Millon \& Davis, 1996; Oldham \& Morris, 1995).

Handler e Meyer (1997), para enfatizar a importância do estudo da personalidade, chamam atenção para três atributos atrelados ao estudo desse construto. Em síntese, eles apontam para a contribuição do estudo da personalidade na formulação de psicodiagnósticos, no entendimento da dinâmica do funcionamento psíquico de um indivíduo e para o desenvolvimento do autoconhecimento. A partir disso, enfatiza-se a importância do estudo da personalidade e de sua avaliação tanto em processos psicoterapêuticos como na realização de pesquisas científicas.

Apesar do consenso em relação à importância do estudo e avaliação da personalidade, observa-se uma clara divergência quanto ao modelo teórico que mais adequadamente seja capaz de definir esse construto (Millon, 1993; Millon, 1986b; Widiger \& Simonsen, 2005). Embora diversidade e pluralismo científico sejam atributos úteis, a acumulação sistematizada de dados a partir de diversas abordagens e a comunicação entre pesquisadores são condições difíceis de serem atingidas em meio a um grande número de conceitos e propostas de avaliação, como se observa no campo de estudo da personalidade (Srivastava, Gosling \& Potter, 2003).

As diferentes abordagens contemporâneas concordam que a personalidade está mais relacionada com o funcionamento psicológico como um todo, e não com fragmentos, por exemplo, apenas $o$ funcionamento mais observável do indivíduo (Millon, 1986b). Os primeiros estudos relacionados à avaliação da personalidade enquanto construto a ser estudado de maneira sistematizada pela psicologia, são freqüentemente atribuídos a Allport, Cattell e Eysenck (Santos, 2006; Pasquali, 1999; Winter, Stewart, John, Klownen \& Duncan, 1998). As concepções apresentadas por esses autores se fundamentaram em filósofos que, centenas de anos antes, estudaram o comportamento do homem e inferiram acerca de seu funcionamento (Matthews, Deary \& Whiteman, 2003; Ortiz, Tello \& Gandara, 2005; Serra, 2006).

\section{Teofrasto e os primóridos do estudo da personalidade}

$\mathrm{Na}$ literatura, considera-se Teofrasto como o precursor do estudo da personalidade (Gosling, 2008), 
Quadro 1

Estilos/Padrões de Funcionamento Segundo Teofrasto

\begin{tabular}{|c|c|c|c|c|}
\hline \multicolumn{5}{|c|}{ Estilos/Padrões de Funcionamento } \\
\hline Dissimulado & Bajulador & Tagarela & Parolo & Complacente \\
\hline Impudente & Parlapatão & Enredador & Descarado & Mesquinho \\
\hline Disparatado & Inoportuno & Intrometido & Estúpido & Autoconvencido \\
\hline Supersticioso & Eterno descontente & Desconfiado & Desmazelado & Inconveniente \\
\hline Pedante & Forreta & Gabarola & Arrogante & Cobarde \\
\hline Ditador & Remoçado & Maledicente & Padrinho do vigarista & Explorador \\
\hline
\end{tabular}

Fonte: os autores com base em Os Caracteres.

que foi um filósofo grego vivente entre 372 a.C. a 287 a.C., e sucessor de Aristóteles. É escassa a literatura atual apresentando e discutindo a contribuição de Teofrasto para o pensamento filosófico de sua época, bem como a influência do filósofo no estudo contemporâneo da personalidade. Especificamente sobre esse último ponto, a ênfase é dada para sua obra denominada Os Caracteres. De acordo com Silva (1999), trata-se de uma das primeiras tentativas documentadas de caracterizar diferentes padrões de comportamentos de seres humanos, que pode ser compreendida como uma tipologia

Para o desenvolvimento de sua obra, e também para propor grupos de pessoas representadas por diferentes caráteres, Teofrasto considerou ambas as perspectivas nomotética e idiográfica, contemporaneamente definidas por Cronbach (1996). Por um lado, cada um dos caractéres, que podem ser entendidos como estilos da personalidade, é descrito de modo a retratar características comuns a determinados grupos de pessoas (perspectiva nomotética). Por um lado, como apontado por Ellis, Abrams e Abrams (2008), Teofrasto utilizou a abordagem idiográfica para descrever estilos prototípicos da personalidade, no sentido de considerar possíveis diferenciações intragrupos em cada um dos estilos que propõe.

Nesse sentido, ainda que na época de Teofrasto não houvesse um estudo sistemático da personalidade, e sequer o conceito desse construto psicológico, é possível enquadrar sua proposta em um modelo tanto nomotético quanto idiográfico. De acordo com Millon e Davis (1996), são poucas as teorias da personalidade contemporâneas capazes de considerar os sistemas nomotético e idiográfico. Os autores utilizam como exemplo uma das propostas atuais mais corroboradas no estudo da personalidade, o modelo dos Cinco Grandes Fatores (CGF) que fundamentalmente parte de uma perspectiva nomotética, na busca por determinar as dimensões gerais que explicam a personalidade humana. A perspectiva nomotética torna-se relevante na medida em que visa determinar as variáveis (dimensões) mais importantes na avaliação da personalidade. Contudo, o modelo CGF não utiliza a noção subjacente ao sistema idiográfico, que está mais voltado para a semelhança entre as pessoas, na tentativa de considerar diversas características ao mesmo tempo. Por isso, modelos que contemplam a perspectiva idiográfica trazem importante contribuição para a prática clínica em termos de entendimento do funcionamento da personalidade (Millon, Millon, Meagher, Grossman \& Ramanath, 2004).

Ainda acerca do que é apresentado em $\underline{\text { Os }}$ Caracteres, há uma seqüência de trinta estilos ou padrões de funcionamento (de I a XXX), contudo, sem que haja uma relação de proximidade entre eles. Por exemplo, o VIII estilo, o enredador, que é aquele que pretende tornar credíveis palavras e fatos falsos, tem pouco a compartilhar com o estilo IX, o descarado, que se caracteriza por desprezar a opinião pública, com vista a benefícios mesquinhos. A Tabela 1 apresenta os trinta estilos propostos por Teofrasto.

Por meio da leitura dos estilos propostos por Teofrasto, observa-se que o filósofo partiu de um sistema classificatório que é atualmente apontado como categórico. Os sistemas categóricos representam os modelos tradicionalmente utilizados na psicologia e psiquiatria para classificação e diagnóstico dos transtornos mentais. De maneira mais ampla, esse sistema busca agrupar as pessoas em diferentes categorias, atribuindo a elas diferenciações qualitativas (Widiger \& Trull, 2007). Um exemplo bastante conhecido no campo da saúde mental, e que utiliza o modelo categórico em sua base, é o Manual Diagnóstico e Estatístico de Transtornos Mentais (DSM), hoje em sua quarta edição (APA, 2003).

Assim, verifica-se uma tendência do ser humano em buscar categorias que agrupem as pessoas de acordo com suas características, o que se reflete na clínica psicológica, nas palavras de Widiger e Samuel (2005, p. 500), “as decisões clínicas são categóricas”. Além disso, as tendências da obra de Teofrasto sugerem que a curiosidade do homem sobre seu próprio funcionamento e, mais especificamente, sobre seu modo de reagir no cotidiano, data de muitos séculos. Indagou Teofrasto no Prefácio de Os Caracteres (p. 49): “(...) como é que, estando a Grécia sob um mesmo céu e recebendo nós, os Gregos, uma 
formação semelhante, conseguimos ser tão diferentes na maneira de ser.". Autores contemporâneos corroboram a idéia de que o ser humano sempre buscou compreender suas próprias características psicológicas (Oldham \& Morris, 1995).

De fato, se por um lado a obra do filósofo imaugurou o que se compreende hoje como a categorização de pessoas que compartilham características em estilos psicológicos, por outro $\underline{\mathrm{Os}}$ Caracteres se limitam a expor por meio de categorias "diversos tipos de personalidade que as caracterizam e o modo como actuam." (p. 49). Nesse sentido a proposta de Teofrasto se assemelha mais aos modelos contemporâneos da personalidade que se focam prioritariamente em dados empíricos, como é o caso do modelo de Cattell e CGF (Goldberg, \& Velicer, 2006). A diferença substancial entre esses últimos e a proposta de Teofrasto enquadra-se no campo metodológico, isto é, o filósofo utilizou-se da observação, e tanto Cattell como pesquisadores do CGF obtiveram seus dados a partir do uso de técnicas estatísticas da psicometria.

Especificamente no que concerne ao método usado por Teofrasto, Gosling (2008, p. 29) aponta que “... embora não dispusesse das ferramentas estatísticas da pesquisa moderna sobre a personalidade, ele tinha os melhores substitutos: um senso agudo de observação, combinado com a habilidade de captar vividamente como certos comportamentos ocorrem em conjunto para gerar tipos de personalidade distintos.”. Gosling afirma, ainda, que Teofrasto buscava observar as pessoas no cotidiano, derivando posteriormente os estilos psicológicos encontrados em sua obra.

Ao lado disso, apesar da relação inequívoca entre a estrutura e o objetivo da obra de Teofrasto com as teorias da personalidade atuais, vale a pena ressaltar que, não raramente, e até mesmo de maneira mais frequente, a literatura aponta para a importância de Hipócrates nas concepções contemporâneas dos estilos da personalidade (Ito \& Guzzo, 2002). Entretanto, a teoria dos humores corporais, utilizada por Hipócrates (e depois por Galeno) para explicar os estados de saúde e doença, contribui de maneira mais direta para o que é entendido hoje como temperamento, mais do que para o estudo da personalidade. Talvez a dificuldade de estabelecer diferenças operacionais entre esses construtos ajude a compreender a confusão que geralmente ocorre. Assim é importante esclarecer que o temperamento, de maneira sucinta, se refere ao potencial biológico subjacente aos comportamentos, observado principalmente em humores e reações emocionais (Millon \& Davis, 1996).

Ainda, cabe ressaltar que, apesar da ênfase dada a Hipócrates em diversos estudos, e o foco do presente texto em Teofrasto, outros filósofos tiveram importância também para as compreensões atuais da personalidade (Millon \& Davis, 1996). Exemplo disso é o filósofo grego Empédocles, com sua proposta de que o mundo funcionaria por meio de quatro elementos: água, ar, fogo e terra. A partir disso, esse filosófo propunha que as pessoas poderiam se diferenciar em relação às suas características mais ou menos salientes.

\section{Considerações Finais}

Este texto teve como objetivo apresentar, de maneira breve, os primeiros passos de estudiosos e filósofos em direção ao que hoje é compreendido como um estudo científico e sistematizado de um dos construtos mais pesquisados dentro da psicologia, a personalidade. Enfatizou-se a importância da obra do grego Teofrasto, Os Caracteres, que constitui um relevante manuscrito que pode ser utilizado como referência histórica para os estudos atuais de tipologia e categorização de diferentes estilos da personalidade.

Vale a pena ressaltar que não foram encontrados estudos buscando relacionar a obra de Teofrasto com perspectivas mais atuais. Nesse sentido, tentou-se aqui não mais que explicitar a estrutura utilizada pelo filósofo que, apesar de muito antes dos pesquisadores contemporâneos, por vezes se assemelha com os trabalhos que são realizados atualmente. Além disso, as derivações das observações de Teofrasto, quais sejam, os estilos por ele propostos, configuram-se como um exemplo de relevância histórica, mas também teórica, já que considera diferentes perspectivas, idiográfica e nomotética, dentro do processo de categorização de estilos psicológicos.

Por fim, é interessante notar, e por vezes pode trazer à reflexão, que apesar da notável evolução do estudo da personalidade em relação à obra de Teofrasto, muito de sua obra ainda persiste, como o modo categórico de sistematizar os diferentes estilos da personalidade. Nesse sentido, torna-se importante voltar a esses antigos manuscritos, a fim de comparálos com o que há de atual e melhor compreendê-los, almejando como conseqüência, o aperfeiçoamento das propostas contemporâneas.

\section{Referências}

Allport, F. H, e Allport, G. W. (1921). Personality Traits: Their Classification and Measurement, Journal of Abnormal and Social Psychology, 16, 6-40.

Allport, G. W. (1937). Personality: A psychological interpretation. New York: Holt.

American Psychological Association. (2003). Manual Diagnóstico e Estatístico de Transtornos Mentais DSM-IV-TR (4 ed.). Porto Alegre: Artmed.

Clapier-Valladon, S. (1988). As Teorias da Personalidade. São Paulo: Martins Fontes.

Cloninger, S. C. (1999). Teorias da Personalidade. São Paulo: Martins Fontes.

Cronbach, L. J. (1996). Fundamentos da testagem psicológica. Porto Alegre: Artes Médicas.

Ellis, A., Abrams, M., \& Abrams, L. Personality Theories: critical perspectives. Sage.

Goldberg, L. R., \& Velicer, W. F. (2006). Principles of 
exploratory factor analysis. In S. Strack (Ed.), Differentiating normal and abnormal personality (pp. 209-237). New York, NY: Springer.

Gosling, S. (2008). Psiu, dê uma espiadinha! Elsevier, São Paulo.

Hall, C. S.; Lindzey, G.; \& Campbell, J. B. (2000) Teorias da Personalidade, $4^{\mathrm{a}}$ ed., Porto Alegre: Artmed.

Handler, L. \& Meyer, G. J. (1997). The importance of teaching and learning personality assessment. In: Handler, L., \& Hilsenroth, M. (eds), Teaching and learning personality assessment, New Jersey: Lawrence Erlbaum Associates.

Ito, P. C. P., \& Guzzo, R. S. L. (2002). Diferenças Individuais: temperamento e personalidade; importância da teoria. Estudos de Psicologia, 19, 1, 91-100.

Matthews, G., Deary, I. J., \& Whiteman, M. C. (2003). Personality Traits. Cambridge University Press, Cambridge.

Mayer, J. D. (2005). A Tale of Two Visions: can a new view of personality help integrate psychology? American Psychologist, 60, (4), 294-307.

Millon, T. (1986a). A Theoretical Derivation of Pathological Personalities. In: Millon, T., \& Klerman, G.L. (eds.), Contemporary Directions in Psychopathology: Toward the DSM-IV. Nova York: Guildford.

Millon, T. (1986b). Personality Prototypes and Their Diagnostic Criteria. In.: Millon, T., \& Klerman, G. L. Contemporary Directions in Psychopathology: Toward the DSM-IV. Nova York: Guildford.

Millon, T. (1993). Personality Disorders: conceptual distinctions and classification issues. In: Costa, P. T., \& Widiger, T. A. Personality Disorders and the Five-Factor Model of Personality, Washington, APA.

Millon, T. \& Davis, R. D. (1996). Disorders of Personality

DSM-IV and Beyond. New Jersey: Wiley.

Millon, T. Millon, C. M., Meagher, S. Grossman, S. \& Ramanath, R. (2004). Personality Disorders in Modern Life. New Jersey: Wiley, 2004.

Oldham, J. M.; \& Morris, L. B. (1995). The New Personality Self-Portrait: Why you think, work, love, and act the way you do. New York :Bantam Books.
Ortiz, M. A. C., Tello, F. P. H., \& Gandara, M. V. B. (2005). Dimensionalidad del cuestionario de los cinco grandes (BFQ-N) en población infantil española. Psicothema, 17, 2, 286-91.

Pasquali, L. (1999). Os Tipos Humanos: a teoria da personalidade. LabPAM, Brasília.

Poulsen, F. (1922). Etruscan Tomb Paintings - Their Subjects And Significance. Oxford, Clarendon Press.

Santos, R. S. (2006). Avaliação da Maturidade PerceptoMotora e Traços de Personalidade. Dissertação de mestrado não publicada, Universidade São Francisco, Itatiba.

Serra, J. D. (2006). Carácter y Temperamento: similitutes y diferencias entre los modelos de personalidade de 7 y 5 factores.

Tese de doutorado não publicada, Universitat de Lleida, Lleida

Silva, M. F. (1999). Introdução, Nota e Tradução de Os Caracteres. Relógio D’Água Editores, Lisboa.

Smith, H. C. (1977). Desenvolvimento da Personalidade. McGraw-Hill, São Paulo.

Srivastava, S., John, O. P., Gosling, S. D., \& Potter, J. (2003). Development of personality in early and middle adulthood: Set like plaster or persistent change? Journal of Personality and Social Psychology, 84, 1041-1053.

Teofrasto. (2010, Agosto, 17). The Characters. Acessado de http://www.eudaemonist.com/biblion/characters

Widiger, T.A., \& Simonsen, E. (2005). The American Psychiatric Association's research agenda for DSM-V. Journal of Personality Disorders, 19, 103-109.

Widiger, T. A., \& Trull, T. J. (2007). Place Tectonics in the Classification of Personality Disorder: shifting to a dimensional model. American Psychologist, 62, 2, 71-83.

Winter, D. G., Stewart, A. J., John, O. P., Klohnen, E. C., \& Duncan, L. E. (1998). Psychological Review, 105, 2, 230-250.

Recebido: $19 / 08 / 2010$ Última Revisão: $15 / 12 / 2010$ Aceite Final: $16 / 12 / 2010$

Sobre os Autores

Lucas de Francisco Carvalho - Mestre e doutorando pelo Programa de Pós Graduação Stricto Sensu em Psicologia, Universidade São Francisco

E-mail: lucas@labape.com.br.

Rodolfo Augusto Matteo Ambiel - Mestre pelo Programa de Pós Graduação Stricto Sensu em Psicologia, Universidade São Francisco

As atividades de pesquisa do primeiro autor que deram origem a esse artigo são financiadas pela FAPESP. 